\title{
Recent hadronic physics results from NA48
}

\section{Cristina Lazzeroni*i}

University of Cambridge, $U K$

E-mail: cristina@hep.phy.cam.ac.uk

This paper contains results of the NA48/1 experiment on neutral hyperon decays, and the first results obtained in the NA48/2 experiment on charged kaon decays. These experiments were carried out in 2002-2004 using different beams specially designed and constructed in the K12 beam line for each of them [1], [2]. Both experiments used the NA48 apparatus [3], updated and optimized accordingly. The accumulated statistics allow high precision study of $K_{S}$, hyperons and charged kaons at a sensitivity not reachable at present in other experiments.

In the fiducial volume $70<E<220 \mathrm{GeV}, 5<Z<50 \mathrm{~m}$ from the final collimator, a large number of $\Xi^{0}$ and $\overline{\Xi^{0}}$ were collected with negligible background: $N\left(\Xi^{0}\right)=\left(2.422 \pm 0.003_{\text {stat }} \pm 0.018_{\text {syst }}\right)$. $10^{9}, N\left(\overline{\Xi^{0}}\right)=\left(2.254 \pm 0.012_{\text {stat }} \pm 0.017_{\text {syst }}\right) \cdot 10^{8}$. The ratio of their fluxes has been measured as a function of the energy while an integrated value is given by: $N\left(\overline{\Xi^{0}}\right) / N\left(\Xi^{0}\right)=\left(9.31 \pm 0.05_{\text {stat }} \pm\right.$ $\left.0.04_{\text {syst }}\right) \cdot 10^{-2}$. In the same fiducial volume were collected samples of: $6316 \Xi^{0} \rightarrow \Sigma^{+} e^{-} \bar{v}_{e}$ events (with the $\Sigma^{+}$decaying into $p \pi^{0}$ ); $515 \overline{\Xi^{0}} \rightarrow \overline{\Sigma^{+}} e^{+} v_{e}$ events; $102 \Xi^{0} \rightarrow \Sigma^{+} \mu^{-} \overline{v_{\mu}}$ events. From these sample the following branching ratios have been measured: $B R\left(\Xi^{0} \rightarrow \Sigma^{+} e^{-} \bar{v}_{e}\right)=(2.51 \pm$ $\left.0.03_{\text {stat }} \pm 0.09_{\text {syst }}\right) 10^{-4} ; B R\left(\overline{\Xi^{0}} \rightarrow \bar{\Sigma}^{+} e^{+} v_{e}\right)=\left(2.57 \pm 0.12(\text { stat })_{-0.09}^{+0.10}(\right.$ syst $\left.)\right) \cdot 10^{-4} ; B R\left(\Xi^{0} \rightarrow\right.$ $\left.\Sigma^{+} \mu^{-} \bar{v}_{\mu}\right)=\left(2.2 \pm 0.3_{\text {stat }} \pm 0.2_{\text {syst }}\right) 10^{-6}$.

The invariant mass of $\pi^{0} \pi^{0}\left(M_{00}\right)$ in $K^{ \pm} \rightarrow \pi^{ \pm} \pi^{0} \pi^{0}$ decays has been studied to search for possible anomalies at the threshold region $M_{00}=2 \cdot m_{\pi^{ \pm}}$and a slope change at the threshold has been detected. Such an anomaly has not been observed in previous experiments. The change of slope observed suggests the presence of a threshold "cusp" effect from the decay $K^{ \pm} \rightarrow \pi^{ \pm} \pi^{+} \pi^{-}$contributing to the $K^{ \pm} \rightarrow \pi^{ \pm} \pi^{0} \pi^{0}$ amplitude through the charge exchange reaction $\pi^{+} \pi^{-} \rightarrow \pi^{0} \pi^{0}$. For the case of $K^{ \pm} \rightarrow \pi^{ \pm} \pi^{0} \pi^{0}$ decay a simple re-scattering model has been proposed[4], describing the decay amplitude as the sum of two terms representing "unperturbed amplitude" and contribution from the $K^{ \pm} \rightarrow \pi^{ \pm} \pi^{+} \pi^{-}$decay amplitude through $\pi^{+} \pi^{-} \rightarrow \pi^{0} \pi^{0}$ charge exchange. The latter contribution is proportional to $a_{x}$, the S-wave $\pi^{+} \pi^{-}$charge exchange scattering length. In the limit of exact isospin symmetry $a_{x}=\left(a_{0}-a_{2}\right) / 3$, where $a_{0}$ and $a_{2}$ are the $\pi \pi$ scattering lengths in the $\mathrm{I}=0$ and $\mathrm{I}=2$ states, respectively. Using a model which takes into account also higher order rescattering[5], a fit to the data has been performed obtaining $\left(a_{0}-a_{2}\right) m_{+}=0.268 \pm 0.0010$ (stat) \pm 0.004 (syst), with an additional $\pm 5 \%$ (theo) from neglecting isospin breaking and radiative corrections.

International Europhysics Conference on High Energy Physics

July 21st - 27th 2005

Lisboa, Portugal

\footnotetext{
*Speaker.

$\dagger$ On behalf of the NA48 collaboration.
} 


\section{1. $\Xi^{0}$ and $\overline{\Xi^{0}}$ semileptonic decays}

The study of hadron beta decays gives important information on the interplay between the weak interaction and the hadron structure determined by the strong interaction. In this context the $\Xi^{0}$ beta decays allow testing both of SU(3) symmetry, via its strong analogy with the well-known neutron beta decay, and of the quark mixing model, via extraction of $V_{u s}$ (the sine of the Cabibbo angle). NA48 collected a sample of $6316 \Xi^{0} \rightarrow \Sigma^{+} e^{-} \bar{v}_{e}$ events (with the $\Sigma^{+}$decaying into $p \pi^{0}$ ) with energy between 70 and $220 \mathrm{GeV}$ and decay vertex between $5 \mathrm{~m}$ and $50 \mathrm{~m}$ from the fi nal collimator (see Fig. 1 left).

With a background of about $2 \%$, a value for the branching ratio has been extracted: $B R\left(\Xi^{0} \rightarrow\right.$ $\left.\Sigma^{+} e^{-} \bar{v}_{e}\right)=\left(2.51 \pm 0.03_{\text {stat }} \pm 0.09_{\text {syst }}\right) 10^{-4}$, where the systematic error is dominated by the trigger effi ciency determination, the geometrical acceptance and the form factors. Including the dependence of the form factors from the transfer momentum and the radiative corrections, the following value for $V_{u s}$ can be extracted from the branching ratio measurement: $V_{u s}=0.208 \pm 0.006_{-0.025}^{+0.030} g_{1} / f_{1}$. The systematic error is largely dominated by the error on the ratio of the form factors $g_{1} / f_{1}$ taken from PDG; the contribution to $V_{u s}$ error coming from the uncertainty on the branching ratio measurement is now comparable with the contribution coming from the uncertainty on the $\Xi^{0}$ lifetime.

In the same data and fi ducial volume, a sample of $515 \overline{\Xi^{0}} \rightarrow \overline{\Sigma^{+}} e^{+} v_{e}$ were also collected with a larger rate of background, (see Fig. 1 right), from which a well consistent value for the branching ratio has been extracted: $B R\left(\overline{\Xi^{0}} \rightarrow \bar{\Sigma}^{+} e^{+} v_{e}\right)=\left(2.57 \pm 0.12(\right.$ stat $){ }_{-0.09}^{+0.10}($ syst $\left.)\right) \cdot 10^{-4}$.

Similar criteria were applied to select $\Xi^{0} \rightarrow \Sigma^{+} \mu^{-} \overline{v_{\mu}}$ decays, except for the requirement applied on the muon detector. A sample of 102 events was selected, with a background of $32 \pm 3.0$ (see Fig. 2 left), from which the measurement of the branching ratio was extracted: $B R\left(\Xi^{0} \rightarrow\right.$ $\left.\Sigma^{+} \mu^{-} \bar{v}_{\mu}\right)=\left(2.2 \pm 0.3_{\text {stat }} \pm 0.2_{\text {syst }}\right) 10^{-6}$. This is the largest sample collected so far of muonic decays.

In the same fi ducial volume a large number of $\Xi^{0} \rightarrow \Lambda \pi^{0}$ and $\overline{\Xi^{0}} \rightarrow \bar{\Lambda} \pi^{0}$ were recorded: $N\left(\Xi^{0}\right)=\left(2.422 \pm 0.003_{\text {stat }} \pm 0.018_{\text {syst }}\right) \cdot 10^{9}, N\left(\overline{\Xi^{0}}\right)=\left(2.254 \pm 0.012_{\text {stat }} \pm 0.017_{\text {syst }}\right) \cdot 10^{8}$ and mainly used for normalization purposes of the various branching ratio measurements. The ratio of their fluxes has been measured as a function of the energy (see Fig. 2 right), while an integrated value is given by: $N\left(\overline{\Xi^{0}}\right) / N\left(\Xi^{0}\right)=\left(9.31 \pm 0.05_{\text {stat }} \pm 0.04_{\text {syst }}\right) \cdot 10^{-2}$.
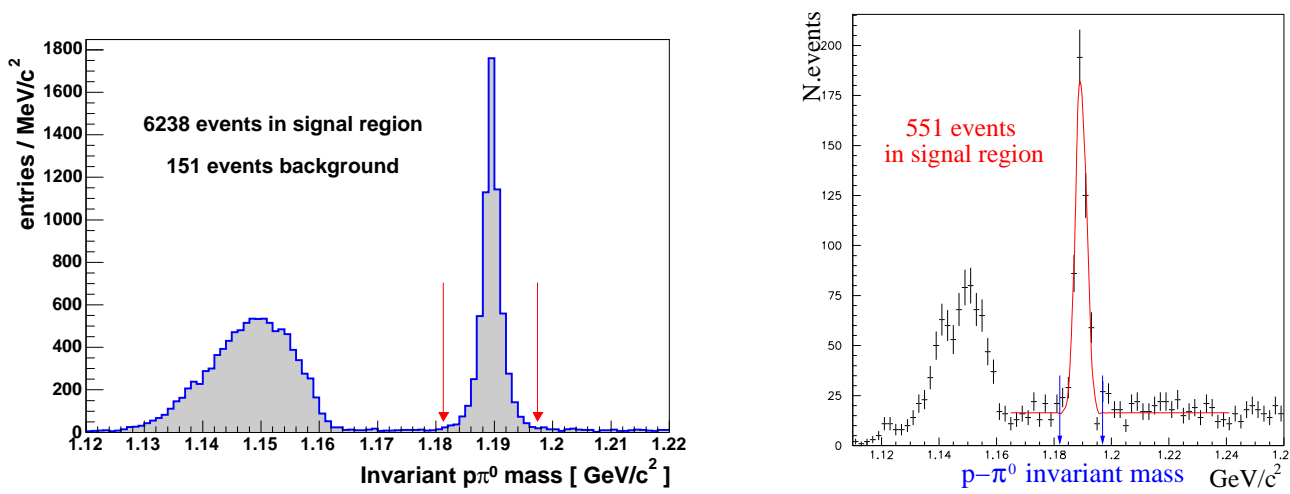

Figure 1: Distribution of $\bar{p} \pi^{0}$ invariant mass for selected events with an electron (left) and a positron (right) 

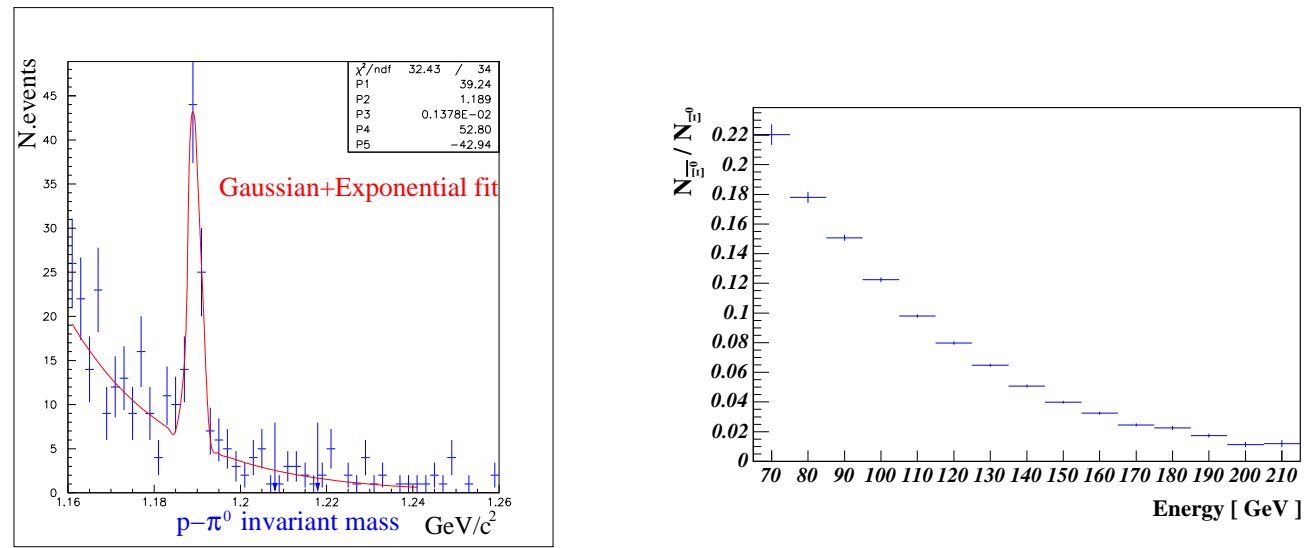

Figure 2: Left: Distribution of $p \pi^{0}$ invariant mass for selected events with a negative muon. Right: The ratio of $\overline{\Xi^{0}}$ and $\Xi^{0}$ fluxes measured in different energy bins.

\section{First observation of a cusp-like structure in $M\left(\pi^{0} \pi^{0}\right)$ from $K^{ \pm} \rightarrow \pi^{ \pm} \pi^{0} \pi^{0}$}

To select $K^{ \pm} \rightarrow \pi^{ \pm} \pi^{0} \pi^{0}$ decays, two neutral pions are identifi ed by proper pairing of four reconstructed gammas in the calorimeter, requiring that both gamma pairs have the closest vertices calculated under the assumption of $\pi^{0} \rightarrow \gamma \gamma$ decays[2]. The invariant mass of $\pi^{0} \pi^{0}$ subsystem $\left(M_{00}\right)$ in $K^{ \pm} \rightarrow \pi^{ \pm} \pi^{0} \pi^{0}$ decays has been studied to search for possible anomaly at the threshold region $M_{00}=2 \cdot m_{\pi^{ \pm}}$.

Fig. 3 (left) shows the distribution of $M_{00}$, without any acceptance correction, for the events in the region close to the threshold $M^{2}\left(\pi^{0} \pi^{0}\right)=4 m_{\pi^{ \pm}}^{2}=0.0779\left(\mathrm{MeV} / \mathrm{c}^{2}\right)^{2}$. The slope change at the threshold is clearly visible. Such an anomaly has not been observed in previous experiments. The acceptance has been studied and shown to be linear in the cusp region.

The sudden change of slope observed in this plot suggests the presence of a threshold "cusp" effect from the decay $K^{ \pm} \rightarrow \pi^{ \pm} \pi^{+} \pi^{-}$contributing to the $K^{ \pm} \rightarrow \pi^{ \pm} \pi^{0} \pi^{0}$ amplitude through the charge exchange reaction $\pi^{+} \pi^{-} \rightarrow \pi^{0} \pi^{0}$. For the case of $K^{ \pm} \rightarrow \pi^{ \pm} \pi^{0} \pi^{0}$ decay Cabibbo [4] has proposed a simple re-scattering model describing the decay amplitude as the sum of two terms representing "unperturbed amplitude" and contribution from the $K^{ \pm} \rightarrow \pi^{ \pm} \pi^{+} \pi^{-}$decay amplitude through $\pi^{+} \pi^{-} \rightarrow \pi^{0} \pi^{0}$ charge exchange. The latter contribution is proportional to $a_{x}$, the S-wave $\pi^{+} \pi^{-}$charge exchange scattering length. In the limit of exact isospin symmetry $a_{x}=\left(a_{0}-a_{2}\right) / 3$, where $a_{0}$ and $a_{2}$ are the $\pi \pi$ scattering lengths in the $\mathrm{I}=0$ and $\mathrm{I}=2$ states respectively.

In this simple re-scattering model [4] there is only one additional parameter $a_{x} m_{+}$, where $m_{+}$ is the charged pion mass. A fit to the $M_{00}^{2}$ distribution in the region $0.074 \leq M_{00}^{2} \leq 0.097\left(\mathrm{GeV} / \mathrm{c}^{2}\right)^{2}$ using $a_{x} m_{+}$, as a free parameter gives $\chi^{2}=420.1$ for 148 degree of freedom. The quality of this fi $t$ is illustrated in Fig. 3 (right,a) which displays the difference between data and best fi t normalized to the data value $(\Delta)$ as a function of $M_{00}$. Recently Cabibbo and Isidori [5] have proposed a more complete model, that takes into account all re-scattering processes in two-loop approximation. In the limit of exact isospin symmetry the five S-wave scattering lengths can be expressed as linear combinations of $a_{0}$ and $a_{2}$. The fit by this model (fig. 3 (right,b)) produce $\not{\chi}=154.8$ for 146 degrees of freedom.

The better fit shown in Fig.3 (right,c) $\left(\chi^{2}=149.1 / 145 d\right.$.f.) is obtained by adding to the model a term describing the expected formation of a $\pi^{+} \pi^{-}$atom ("pionium") decaying into $\pi^{0} \pi^{0}$ 

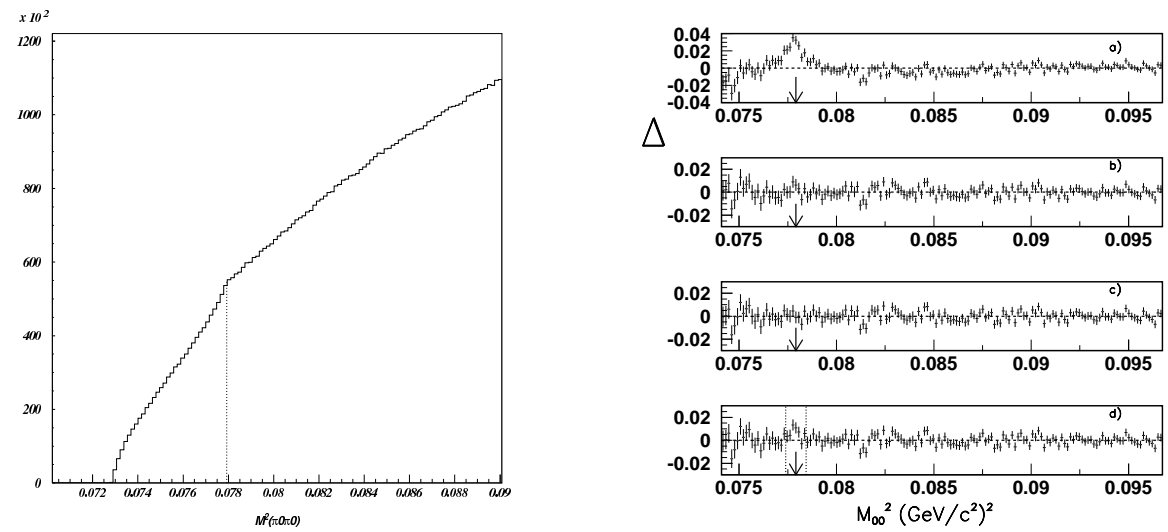

$\left(G e V / c^{2}\right)^{2}$

Figure 3: Left: The $M^{2}\left(\pi^{0} \pi^{0}\right)$ distribution in the region around the threshold. Right:Difference between data and best fits, normalized to the data value, in different versions of the model.

at $M_{00}=2 m_{+}$. The fi t value for the rate of $K^{ \pm} \rightarrow \pi^{ \pm}+$pionium decays is $(1.61 \pm 0.66) \cdot 10^{-5}$, which is in agreement with the predicted value $\sim 0.8 \cdot 10^{-5}[6]$.

The model used do not include radiative corrections, which are particularly important near $M_{00}=2 m_{+}$, and contribute to the formation of the pionium. For this reason a group of seven consecutive bins centered at $M_{00}=2 m_{+}$have been excluded from the fi nal fi t shown in fig. 3(right,d) $\left(\chi^{2} / d . f .=145.5 / 139\right)$.

Study of systematics shows that no corrections are needed. However, the corresponding uncertainty were taken into account. For measured $\left(a_{0}-a_{2}\right) m_{+}$parameter the major ones are the following: acceptance calculation $( \pm 0.001)$; trigger effi ciency $( \pm 0.001)$; fi t interval $( \pm 0.0025)$; $\pi-\gamma$ minimum distance at the $\operatorname{LKr}( \pm 0.002)$; calorimeter resolution and non-linearity $(\leq 0.001)$. Using the model that takes into account higher order rescattering and using the above estimate of systematic uncertainty, the following result is obtained: $\left(a_{0}-a_{2}\right) m_{+}=0.268 \pm 0.0010$ (stat $) \pm$ 0.004 (syst) \pm 0.013 (theo), where the theoretical uncertainty comes from neglecting isospin breaking and radiative corrections. This value is in good agreement with the results on $\left(a_{0}-a_{2}\right) \cdot m_{+}$ obtained in the E865 BNL [7] and Dirac [8] experiments.

\section{References}

[1] NA48/1 Status Report SPSC/M670, CERN/SPSC 2001-029 (2001).

[2] NA48/2 Status Report SPSC/M724, CERN/SPSC 2004-030 (2004).

[3] J. R. Batley et al. Phys.Lett. B 544 (2002) 97.

[4] N. Cabibbo, Phys.Lett. 93 (2004) 121801.

[5] N. Cabibbo and G. Isidori, JHEP 503 (2005) 021.

[6] Z.K. Silagadze, JETP Letters 60(1994)689.

[7] S. Pislak et al. Phys.Rev. D67(2003)072004.

[8] B. Adeva et al., hep-ex/0504044(2005). 